ORIGINAL

\title{
EFECTO DEL DESMANE Y REMOCIÓN DE DEDOS SOBRE LA CALIDAD Y PRODUCCIÓN DEL BANANO
}

\section{EFFECT OF DEHANDING AND REMOVAL OF FINGERS ON THE QUALITY AND PRODUCTION OF BANANA TREE}

\author{
José L. Barrera', Carlos F. Salazar², Karen L. Arrieta² \\ Recibido para evaluación: Junio 21 de 2010 - Aceptado para publicación: Noviembre 30 de 2010
}

\begin{abstract}
RESUMEN
El trabajo se realizó en el municipio de Turbo, Antioquia, con el objetivo de evaluar el efecto de la remoción de manos y dedos laterales del racimo sobre los componentes de calidad y producción del banano. Se evaluaron cuatro tratamientos, con 70 repeticiones por tratamiento, distribuidos completamente al azar. Los tratamientos fueron T1 (eliminación de la mano falsa que presentan flores femeninas y masculinas simultáneamente en el mismo nódulo floral + las 3 manos anteriores de arriba hacia abajo que poseen solo flores femeninas, sin remoción de laterales en todas las manos femeninas), T2 (eliminación de la mano Falsa + las 3 manos femeninas anteriores, con remoción de un lateral a la izquierda y uno a la derecha, en las tres primeras manos superiores), T3 (Eliminación de la mano Falsa $+2^{112}$ manos femeninas anteriores, con remoción de un lateral a la izquierda y dos a la derecha, en todas las manos femeninas del racimo), T4 (Eliminación de la mano Falsa $+1^{112}$, mano femenina anterior, con remoción de un lateral a la izquierda y dos a la derecha, en todas las manos). Se encontró que los tratamientos no afectaron significativamente el peso de racimo. Las variables largo y grado de la fruta en la segunda mano basal y última mano apical mostraron efectos significativos favorables sobre estos parámetros.
\end{abstract}

Palabras clave: Podas, mano falsa, cosecha, calidad, remoción, Musa AAA

\begin{abstract}
The work was conducted in the municipality of Turbo, Antioquia, in order to evaluate the effect of the removal of hands and fingers on the side of cluster quality components and banana production. Four treatments were evaluated with 70 replicates per treatment, randomly distributed. The treatments were T1 (removal of the fake hand with male and female flowers occurring simultaneously on the same floral node + the 3 previous hands up and down that has only female flowers, without removal of laterals in all female hands), T2 (Elimination of
\end{abstract}

${ }^{1}$ Ingeniero Agrónomo MSc. Profesor asociado, Facultad de Ciencias Agrícolas, Universidad de Córdoba. Montería, Colombia. E-mail: jbarrera11@sinu.unicordoba.edu.co

${ }^{2}$ Ingeniero Agrónomo. Estudiante de Maestría en Ciencias Agronómicas, Facultad de Ciencias Agrícolas Universidad de Córdoba, Montería, Colombia 
false female hand + the 3 previous female hands, with removal of one lateral on the left side and one on the right side in the first three superior hands), T3 (Elimination of false hand $+2^{112}$ previous female hands, with removal of $s$ one lateral on the left side and two on the right side over all feminine hands of the bunch), T4 (Elimination of false hand $+1^{112}$ previous female hand, with removal of one lateral on the left side and two on the right side, in every hands). It was found that the treatments did not significantly affect bunch weight. Length and variable degree of fruit on second basal hand and final apical hand showed significant beneficial effect on these parameters.

Keywords: Pruning, fake hand, harvesting, quality, removal, Musa AAA

\section{INTRODUCCIÓN}

Como consecuencia de las exigencias de los mercados de exportación con relación a la longitud mínima de dedos y a un diámetro mínimo, los técnicos bananeros y cultivadores idearon la poda de las manos interiores, cuyos dedos en forma general no alcanzan la longitud ni el grado deseado. A causa de la alta incidencia de Sigatoka negra en muchos países, la reducción de la superficie fotosintética es una realidad, con la consecuente disminución en el Ilenado de almidones y por tanto del grado. En estas circunstancias la poda de manos inferiores y aun superiores parece ser una opción aceptable, lo mismo sucede en regiones donde el llenado de almidones, es lento, consecuencia del clima, tal es el caso de Ecuador en los meses de mayo a agosto; debido a lo anterior, la práctica de desmane se ha convertido en una herramienta muy útil para manejar las dimensiones de la fruta (Asociación Naturland 2001).

El desmane es una práctica cultural que consiste en eliminar o podar una o varias manos durante la labor de protección de fruta. Se eliminan las manos apicales que generalmente no cumplen con las especificaciones del largo del dedo exigidas en los mercados para bananos de exportación (Sierra 1993). De otra forma se entiende la práctica del desmane como la eliminación de varias manos distales, para que la materia seca no utilizable comercialmente se distribuya entre las manos que permanecen en el racimo, que deben aumentar su tamaño (Rodríguez et al. 1988).

De esta manera a la práctica de desmane se le atribuye un aumento en el peso del racimo y obtención de la madurez fisiológica más rápidamente e incremento en la longitud y diámetro de los dedos (Sierra 1993). Irizarry et al. (1991) en Puerto Rico evaluaron el efecto del desmane manos apicales, dejando 4, 5 y 6 manos basales y se reportaron ganancias en el peso de la fruta con cuatro manos. Esta reacción ocurre en respuesta a una disminución en la tasa respiratoria por parte de la planta puesto que se elimina materia seca no utilizable comercialmente, lo que se refleja en una redistribución de los asimilados, aumentando el tamaño de las manos presentes en el racimo.

En el racimo del plátano 'Dominico hartón' (Musa cv. AAB), las manos ubicadas hacia la base del mismo son a menudo de mayor tamaño que las localizadas en la parte terminal (Aristizábal 1995) y, generalmente, esas últimas manos se desechan o en el mejor de los casos se venden como plátano de tercera. Lo anterior significa que de cada racimo producido, al 
menos dos o tres manos no cumplen con los estándares de calidad que se manejan en los mercados especializados, lo cual representa menos ingresos para el productor (Quintero y Aristizábal 2003).

Se han realizado diversos ensayos (Bald et al. 1999a; Bald et al. 1999b; Vargas y Blanco 2000; Vargas 2002), que señalan que la práctica de la poda de manos es inútil, porque no se recupera la pérdida de peso del racimo, aunque si se obtiene un mayor grosor y longitud en las manos inferiores, lo cual es de gran importancia para mejorar la calidad de la fruta para el mercado internacional. Sierra (1993), reporta resultados muy positivos en banano, debido al aumento en grado, longitud y apariencia de la fruta, considera dicha práctica de gran importancia para el manejo de las dimensiones (grado y longitud) de la fruta para exportación en Urabá Antioqueño.

En los últimos años en asocio con el desmane se ha venido practicando la remoción selectiva de dedos laterales (desdede) reflejando aparentemente resultados positivos, sin embargo no se cuenta con reportes sobre la integración de estas dos prácticas y su efecto sobre las características productivas del racimo.

Con el objetivo de generar una primera información de acercamiento con datos específicos que se aproximen al máximo a las condiciones de campo (incluyendo la cosecha), se planteó la presente investigación ante la necesidad de realizar la evaluación de la labor de desmane asociada a la remoción de dedos del racimo, para comprobar su influencia sobre la mejora en la producción de fruta tipo exportación.

\section{MATERIALES Y MÉTODOS}

El ensayo se realizó durante el primer y segundo semestre del año 2008, en el municipio de Turbo, Zona de Urabá, departamento de Antioquia, en la finca Agripina.

La realización de las labores aplicadas (desmanes o podas de manos y eliminación de dedos laterales) para cada tratamiento se aplicaron cuando el racimo mostró las características ideales para la ejecución de la labor, o sea al momento del desflore (Cambio de color de la flor) y la presencia de la mano falsa (Presencia de dedos masculinos y femeninos en el mismo nódulo floral); este ensayo se desarrolló de forma consecutiva durante cuatro semanas hasta finalizar el número total de plantas seleccionadas por tratamiento. Los tratamientos se distribuyeron en el campo en un diseño de bloques completos al azar. Estos consistieron en las siguientes labores: T1 (eliminación de la mano falsa que presentan flores femeninas y masculinas simultáneamente en el mismo nódulo floral + las 3 manos anteriores de arriba hacia abajo que posen solo flores femeninas, sin remoción de laterales en todas las manos femeninas), T2 (eliminación de la mano Falsa + las 3 manos femeninas anteriores, con remoción de un lateral a la izquierda y uno a la derecha, en las tres primeras manos superiores), T3 (Eliminación de la mano Falsa $+2^{112}$ manos femeninas anteriores, con remoción de un lateral a la izquierda y dos a la derecha, en todas las manos femeninas del racimo), T4 (Eliminación de la mano Falsa $+1^{112}$, mano femenina anterior, con remoción de un lateral a la izquierda y dos a la derecha, en todas las manos); distribuidos en un diseño al azar, 
con cuatro tratamientos y 70 repeticiones por tratamiento del clon Gran enano Musa AAA Simmonds. Como parámetro para la cosecha se utilizó la calibración del grosor del dedo a 13 líneas (una línea equivale a 10,3 mm), cosechando racimos entre 11 y 12 semanas de edad después de floración. Las variables evaluadas fueron: peso del racimo $(\mathrm{Kg})$, longitud del dedo central de la segunda mano basal y última mano apical $(\mathrm{cm})$ y grado del dedo central de la segunda mano basal y última mano apical (mm), cicatrices de crecimiento, dedos deformes y mancha de madurez. Para el análisis estadístico de los datos se utilizó el programa estadístico SAS y la prueba de rango múltiple de Tukey versión 9,0 con una probabilidad de 0,05.

\section{RESULTADOS Y DISCUSIÓN}

\section{Peso del racimo}

No se reportaron efectos significativos para esta variable (Tabla 1). La permanencia del mayor número de manos no se reflejó en peso bruto del racimo, esto se debe principalmente a que las manos adicionales no compensaron la pérdida del peso total a causa de la remoción de dedos laterales, fisiológicamente este comportamiento se debe a que la mayor presencia de dedos influye negativamente en la relación fuente vertedero, debido a que los asimilados translocados al racimo tienen que redistribuirse en mayor número de vertederos generando mayor gasto energético y menos acumulación de azucares en los sumideros (Azcon y Bieto 2008).

En diferentes estudios donde se ha evaluado la poda de manos sin asociarse a la remoción de dedos laterales (Bald et al. 1999a; Vargas 2002; Aristizábal 2004 y Barrera et al. 2007) reportan que el peso del racimo disminuye a medida que aumenta la intensidad del desmane, sin embargo, se logró mejorar la calidad de los frutos. En contraste, en esta investigación se encontró que los mayores valores numéricos obtenidos de peso de racimo fueron aquellos en los que se realizó mayor intensidad de desmane, demostrando que la práctica de desmane en el cultivo de banano bajo las condiciones del Urabá favorece la relación fuente vertedero, manifestada por AsconBieto (2008). Estos resultados coinciden con lo reportado por Soto y Calvo (1987), quienes

Tabla 1. Efecto de cuatro tratamientos de desmane y remoción de dedos sobre el peso del racimo de banano

Tratamientos

T1 (mano falsa +3 , sin remoción de laterales)

T2 (Falsa +3 con remoción de un lateral a la izquierda y uno a la derecha, en las tres primeras manos)

T3 (Falsa $+2^{112}$, con remoción de un lateral a la izquierda y dos a la derecha, en todas las manos)

T4 (Falsa $+1^{1 \backslash 2}$, con remoción de un lateral a la izquierda y dos a la derecha, en todas las manos)
Peso del racimo $(\mathrm{kg})$

$28,02^{\mathrm{a}}$

$27,83^{\mathrm{a}}$

$27,72^{\mathrm{a}}$

$26,47^{\mathrm{a}}$

*Medias con letras iguales no difieren estadísticamente, según la prueba de Tukey $(P<0,05)$. 
afirman que la práctica de la poda de manos en banano puede incrementar el preso del racimo en 1,5 a 2,7 Kg por racimo.

\section{Grosor de la fruta}

Se reportaron efectos altamente significativos entre tratamientos para la variable grosor de la fruta de la segunda mano basal y la última mano apical, respectivamente. La prueba de separación de medias Tukey 0,05 muestra que en los tratamientos T2, T3 y T4, en los cuales se realizó mayor intensidad en la remoción de dedos laterales y menor intensidad en la eliminación de manos, se presentaron los mayores valores promedios del grado de la fruta en comparación con el T1 donde no se realizó eliminación de dedos laterales (Tabla 2). Esto muestra que la remoción de dedos laterales contribuye en la redistribución de asimilados en el racimo órgano vertedero, observando mayor ganancia en grado a la fruta.

Vargas y Blanco (2002) y Barrera et al. (2007) reportaron que para el plátano son pocos los efectos del desmane sobre el grosor de la fruta cuando los racimos son sometidos a diferentes intensidades de desmane. En contraste, Soto y Calvo (1987) reportan que en banano los tratamientos donde se realizó desmane presentaron incrementos en el diámetro de los dedos, logrando un diámetro óptimo para exportación, los resultados obtenidos demuestran que la práctica de eliminación de dedos laterales y poda de mano bajo las condiciones ecológicas de Urabá mejoran ostensiblemente la redistribución de asimilados, pero la eliminación de dedos laterales contribuye en virtud de que se elimina la presión que éstos ejercen sobre los dedos centrales, favoreciendo la acumulación de almidones en el fruto, y contribuyendo en la ganancia de diámetros del fruto.

\section{Longitud del fruto de segunda mano basal y última mano apical}

Solo se observaron efectos significativos de los tratamientos para la variable longitud de la última mano apical favoreciendo positivamente esta variable. En la tabla 3 se observa que los tratamientos T2 y T3 obtuvieron los mayores longitudes del dedo apical, puesto que fueron los tratamientos donde la intensidad de desmane fue mayor, permitiendo así mayor alargamiento de los dedos, lo que demuestra que hay más aprovechamiento de la fruta. Demostrando que la práctica del desmane

Tabla 2. Efecto de cuatro tratamientos de desmane y remoción de dedos sobre el grosor de la fruta del banano

\begin{tabular}{ccc}
\hline Tratamientos & $\begin{array}{c}\text { Calibración de la segunda mano } \\
\text { basal }(\mathbf{m m}) .\end{array}$ & $\begin{array}{c}\text { Calibración de la última mano } \\
\text { apical }(\mathbf{m m}) .\end{array}$ \\
\hline T1 & $8,92 \mathrm{~b}$ & $6,30 \mathrm{~b}$ \\
T2 & $9,37 \mathrm{ab}$ & $7,08 \mathrm{a}$ \\
T3 & $9,88 \mathrm{a}$ & $7,12 \mathrm{a}$ \\
T4 & $9,88 \mathrm{a}$ & $6,94 \mathrm{a}$ \\
Significancia & 0,0001 & 0,001 \\
Cv & 3,31 & 4,22 \\
Media & 9,51 & 5,08 \\
\hline
\end{tabular}

*Medias con letras iguales no difieren estadísticamente, según la prueba de Tukey $(P<0,05)$ 
Tabla 3. Efecto de cuatro tratamiento de desmane y remoción de dedos sobre la longitud de la fruta del banano

\begin{tabular}{ccc}
\hline Tratamientos & $\begin{array}{c}\text { Longitud de la segunda mano } \\
\text { basal } \mathbf{( c m})\end{array}$ & $\begin{array}{c}\text { Longitud de la última mano } \\
\text { apical }(\mathbf{c m}) .\end{array}$ \\
\hline T1 & $22,87 \mathrm{a}$ & $18,87 \mathrm{~b}$ \\
T2 & $23,62 \mathrm{a}$ & $19,80 \mathrm{a}$ \\
T3 & $23,62 \mathrm{a}$ & $19,35 \mathrm{ab}$ \\
T4 & $23,40 \mathrm{a}$ & $18,50 \mathrm{C}$ \\
Significancia & 0,20 & 0,0001 \\
CV & 6,12 & 8,40 \\
Media & 23,37 & 19,13 \\
\hline
\end{tabular}

*Medias con letras iguales no difieren estadísticamente, según la prueba de Tukey $(\mathrm{P}<0,05)$

favorece positivamente la calidad del fruto en virtud a que mejora la relación fuentevertedero, incrementando la acumulación de asimilados en el fruto.

Vargas (2002), reporta que la labor de desmane no afecta significativamente la longitud externa del fruto. Soto y Calvo (1987) y Delgado et al. (2003) encontraron diferencias en la longitud de los dedos, observándose mayor longitud en los tratamientos donde se realizó desmane, corroborándose lo anteriormente explicado.

\section{Calidad}

Otras variables que se vieron afectadas por los tratamientos en el desarrollo del ensayo fueron: Cicatrices de crecimiento: Los mayores porcentajes de participación de la merma por cicatrices de crecimiento se presentaron en el T1 y el T2 con 5,5\% y 5,1\%, respectivamente. Los tratamientos T3 y T4 presentaron menor incidencia de cicatrices de crecimiento $2, .22 \%$ y $1,46 \%$, respectivamente (Figura 1 ). Estos resultados demuestran que al eliminar los dedos laterales se reduce la posibilidad

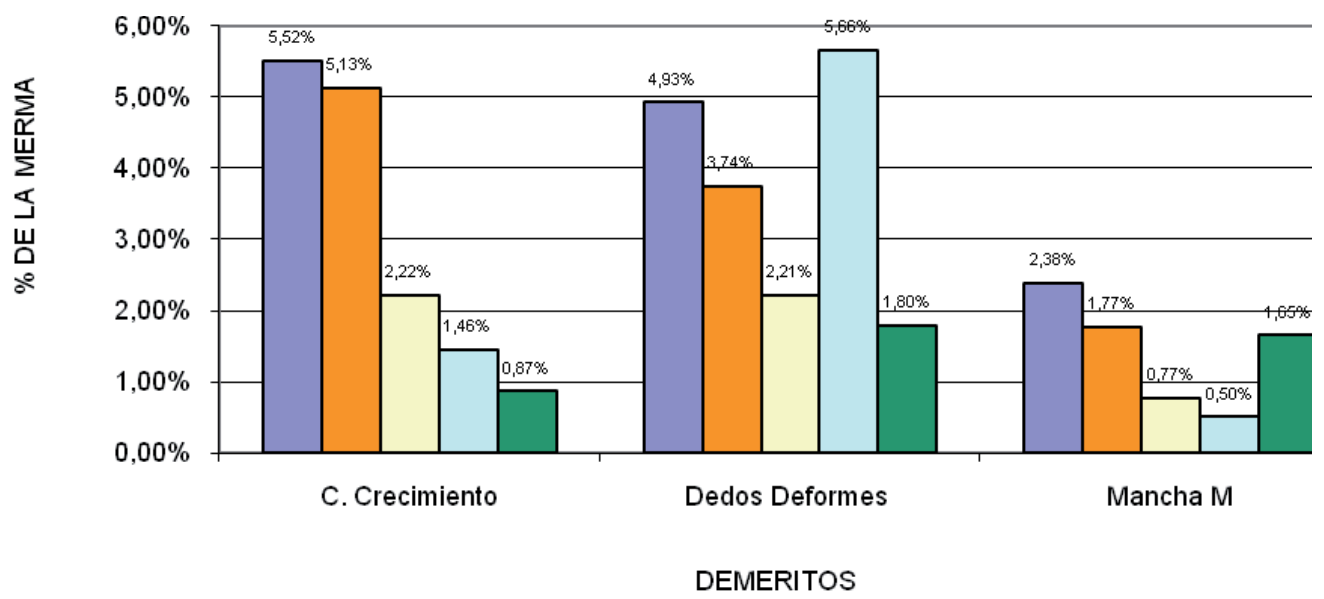

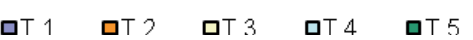

Figura 1. Porcentaje Promedio de Participación de Cicatrices de Crecimiento, Dedos Deformes y Mancha de Madurez en la Merma de los Tratamientos Evaluados 
de que las manos inferiores tengan contacto o rocen con las manos superiores del racimo, causando el daño de punta vieja (cicatriz seca) y/o daño de la hoja (Cicatriz viva) (Asociación Naturland 2001)

Dedos deformes: En los tratamientos T1 y T4 se presentaron los mayores porcentajes de dedos deformes con $4,93 \%$ y $5,6 \%$, respectivamente (Figura 1). El alto porcentaje de dedos deformes en el T4 probablemente se presentó debido a la mayor cantidad de manos y menor cantidad de dedos por mano, puesto que se eliminaron cuatro dedos laterales ( 1 a la izquierda y 3 a la derecha), creciendo separados uno del otro, expandiéndose con más facilidad y por lo tanto haciendo que éstos se deformen. El T3 presentó el menor porcentaje de dedos deformes $(2,21 \%)$, lo que indica que la remoción de 1 dedo lateral a la izquierda y 2 a la derecha, reduce considerablemente la cantidad de dedos deformes, presentando una buena distribución de los dedos en las manos del racimo, logrando un mayor aprovechamiento de la fruta. Soto y Calvo (1987) reportan que en los tratamientos donde se realizó desmane disminuyó considerablemente la curvatura de los dedos. Vargas (2002), encontró que el índice de la curvatura de la fruta no se diferencia entre diferentes intensidades de desmane, Barrera, et al. (2007) y Aristizábal (2004) reportan que el desmane no influyó en los parámetros de producción del racimo, pero aumentó el porcentaje de fruta aprovechable para la exportación, porque mejora los parámetros de calidad. Rodríguez, et al. (1988) también afirman que la práctica del desmane en el cultivo de plátano mejora la calidad de la fruta, pero disminuyen el peso del racimo.
Mancha de madurez: este demérito mostró una mayor participación porcentual promedio dentro de la merma en los tratamientos T1 (2,38\%) y T2 (1,77\%). Se observó disminución de la mancha de madurez en los tratamientos donde se removieron mayor cantidad de dedos laterales T3 y T4 con $0,77 \%$ y $0,5 \%$, respectivamente. Estos resultados demuestran que el espacio generado por la eliminación de dedos laterales entre los dedos centrales de las manos femeninas, contribuyen positivamente a disminuir la presencia de la enfermedad en los frutos.

\section{CONCLUSIONES}

Las labores de poda de manos y remoción de dedos laterales no afecta significativamente el peso del racimo.

Las dimensiones de la fruta (largo y grosor) se ven favorecidas significativamente por las labores de desmane y remoción de dedos laterales.

El desmane asociado al desdede disminuye el porcentaje de la merma, puesto que reduce los deméritos: cicatrices de crecimiento, dedos deformes y mancha de madurez.

\section{REFERENCIAS}

Asociación Naturland. 2001. Agricultura Orgánica en el Trópico y Subtrópico, Guías de 18 cultivos, Banano, Capitulo Empaque de la fruta- $2^{a}$ edición, p 852

Aristizábal L.M. 1995. Componentes del rendimiento en plátano (Musa $\mathrm{AAB} \mathrm{cv}$ 'Dominico hartón'). Revista Universidad de Caldas 15(1-2): 65-75. 
Aristizábal, M, 2004. Efectos del desmane y la distancia de siembra sobre las características productivas del plátano 'FHIA-20'. Infomusa 13 (1), p 9-12.

Azcon-Bieto, j. \& m. Talon. 2008. Fundamentos de fisiología vegetal. Interamericana Mc graw hill, Madrid, p522 .

Bald, A., Sandoval, J. y Target, F. 1999a. Effect of the desmane on the dimensions of the fruit and the weight of the cluster of banana tree (Musa AAA, cv. "Valery"). Corbana 24 (51), p 85 - 91.

Bald, A., Sandoval, J. y Target, F. 1999b. Effect of desmane on the quality of the cluster in banana cv. "False Horn" (Musa AAB) dwarfed and semigiant. Corbana 25 (52), p 129 - 142 .

Barrera, J., Vergara, D. y Marin, O. 2007. Contribución del desmane y el embolse del racimo a la producción y calidad del platano harton. Agronomia. 15(1), p39-44.

Delgado, E., Gonzalez, O., Moreno, N y Romero, D. 2003. Efecto del desmane sobre el peso del racimo y las dimensiones del fruto del híbrido de plátano FHIA 21 (Musa AAAB). Bioagro, ene., vol.15, no.1, p.17-22. ISSN 1316-3361.

Irizarry, H., E. Rivera, A. Krikorian y J. Rodríguez. 1991. Proper bunch management of the French-type superplantain (Musa acuminata $\times$ M balbisiana, AAB) in Puerto Rico. Journal of Agriculture of the University of Puerto Rico 75(2):163-171.
Quintero S. y M. Aristizábal. 2003. Efecto del desmane sobre las características productivas de 'Dominico hartón' y 'Africa' en Colombia L. Revista Internacional sobre Bananos y Plátanos Info musa Vol. 12 No.1.

\section{Rodríguez J.A., H. Irizarry \& E. Rivera.} 1988. Efecto de la poda de manos en el rendimiento y calidad de las frutas del plátano (Musa acuminata $x$ Musa balbisiana, AAB). in ACORBAT. Memorias VIII reunión (R. Jaramillo, A. Restrepo, R Bayona, eds). AUGURA, Medellín, Colombia, p. 537-541.

Soto, B., Calvo, J. 1987. Efecto del desmane en la calidad del fruto de banano, en el clon Gran enano Musa AAA, subgrupo Cavendish, Universidad de Costa Rica. En: Memorias VII Reunión ACORBAT. San José, Costa Rica, p 311- 315.

Sierra, L. 1993. El cultivo del banano producción y comercio. Medellín, Colombia, p 679.

Vargas Calvo, A. y Blanco F. 2000. Methodological considerations in the evaluation of banana bunch trimming (Musa AAA, cv. 'Valery'). Infomusa 9 (2), p $19-21$.

Vargas Calvo, A. 2002. High dehanding Intensity in banana (Musa AAA, cvs. "Grande Naine" and "Williams") and their effect on bunch weight and fruit dimensions. Corbana 28 (55), p 27 - 42. 